\title{
Ökonomische Konsequenzen der Verbissprobleme an der Rigi-Nordlehne
}

Nora Gasser Monika Frehner Jürg Zinggeler Roland Olschewski

\author{
Gadola + Partner GmbH, Rabius (CH)* \\ Waldbau und Waldmanagement, Institut für Terrestrische Ökosysteme, ETH Zürich (CH) \\ Jagdverwaltung Kanton Zürich $(\mathrm{CH})$ \\ Eidgenössische Forschungsanstalt für Wald, Schnee und Landschaft $(\mathrm{CH})$
}

\section{Economic consequences of browsing problems on Rigi-Nordlehne}

\begin{abstract}
Stand regeneration with suitable tree species plays a key role in the management of protection forests. This is complicated in many regions in Switzerland due to browsing damage caused by game animals. In particular, the regeneration of the valuable Abies alba species is regionally heavily impaired. This can entail considerable expenses for the support of stand regeneration and compensation of lost protective effects. Using the RigiNordlehne in Switzerland as test location, the following three questions were studied with the help of an analysis of cost-effectiveness and based on the Rigfor forest development model: 1) What costs are to be expected over the next 50 years for protection measures against browsing damage, 2) what would be the cost of a reduction of the game population size and 3) what measures are necessary for the replacement of the protective effects against avalanches and what are the respective costs? The study resulted in cost estimations for technical measures against browsing damage, game regulation and the combination of these two strategies. In addition, costs for the replacement of lost protective effects related to snow movements were estimated. The study shows, that the high pressure of browsing damage at the Rigi-Nordlehne causes forest management problems as well as economic disadvantages. Their amount is 3 million to 6.6 million Swiss francs within 50 years depending on the measures taken. Thus, the results obtained by the study at the Rigi-Nordlehne provide a new argument in the discussion of regeneration in protection forests, which in Switzerland for many years has mainly been lead based on silviculture and game ecology issues.
\end{abstract}

Keywords: browsing damage, protection forest, cost-effectiveness analysis doi: $10.3188 /$ szf.2011.0364

*Casa da scola, CH-7172 Rabius, E-Mail gadola.gasser@bluewin.ch

I m Rahmen der «Strategie Naturgefahren» der nationalen Plattform Naturgefahren (Planat) wird angestrebt, mit den eingesetzten finanziellen Mitteln ein Maximum an Risikoreduktion zu erreichen (Planat 2005). Dabei spielt der Schutzwald eine wichtige Rolle: In vielen Fällen bietet er einen umfassenden und kosteneffizienten Schutz gegen Lawinen, Steinschlag, Rutschungen und Murgänge. Auch für die Schutzwaldpflege gilt der Grundsatz, dass mit den vorgenommenen Eingriffen die bestmögliche Schutzwirkung erreicht werden soll. Anleitung dazu gibt die Vollzugshilfe des Bundesamts für Umwelt (Bafu) «Nachhaltigkeit und Erfolgskontrolle im Schutzwald» (NaiS; Frehner et al 2005). In Bezug auf die Nachhaltigkeit eines Schutzwaldes spielt die Frage nach der langfristigen Stabilität eine Schlüsselrolle. Um diese gewährleisten zu können, braucht es zum richtigen Zeitpunkt eine ausreichende und entwicklungsfähige Verjüngung. Besonders in hohen Lagen gestaltet sich die Verjüngung von Waldbeständen aufgrund der schwierigen klimatischen
Bedingungen oft nicht einfach. Zusätzlich wird die Verjüngung in den Schweizer Alpen und Voralpen häufig durch hohen Verbissdruck erschwert (z.B. Brassel \& Brändli 1999). Insbesondere die Verjüngung der Weisstanne, die hervorragende waldbauliche Eigenschaften aufweist, wird wildbedingt in vielen Gebieten der Schweiz verlangsamt und teilweise verhindert (z.B. Odermatt et al 1999). Gemäss NaiS ist für eine effiziente Schutzwaldbewirtschaftung in Tannen-Buchen- und Tannen-Fichten-Wäldern ein bestimmter Tannenanteil aber unbedingt erforderlich (Frehner et al 2005).

Die Wald-Wild-Diskussion wurde bisher in der Schweiz hauptsächlich mit waldbaulichen und wildtierökologischen Argumenten geführt. Dies unter anderem deshalb, weil es sowohl bezüglich wildtierökologischer als auch waldbaulicher Fragen häufig schwierig ist, Argumente mit Zahlen und Fakten zu belegen. So kann denn auch selten aufgezeigt werden, was ein hoher Verbissdruck für den Waldeigentümer aus ökonomischer Sicht bedeutet. 


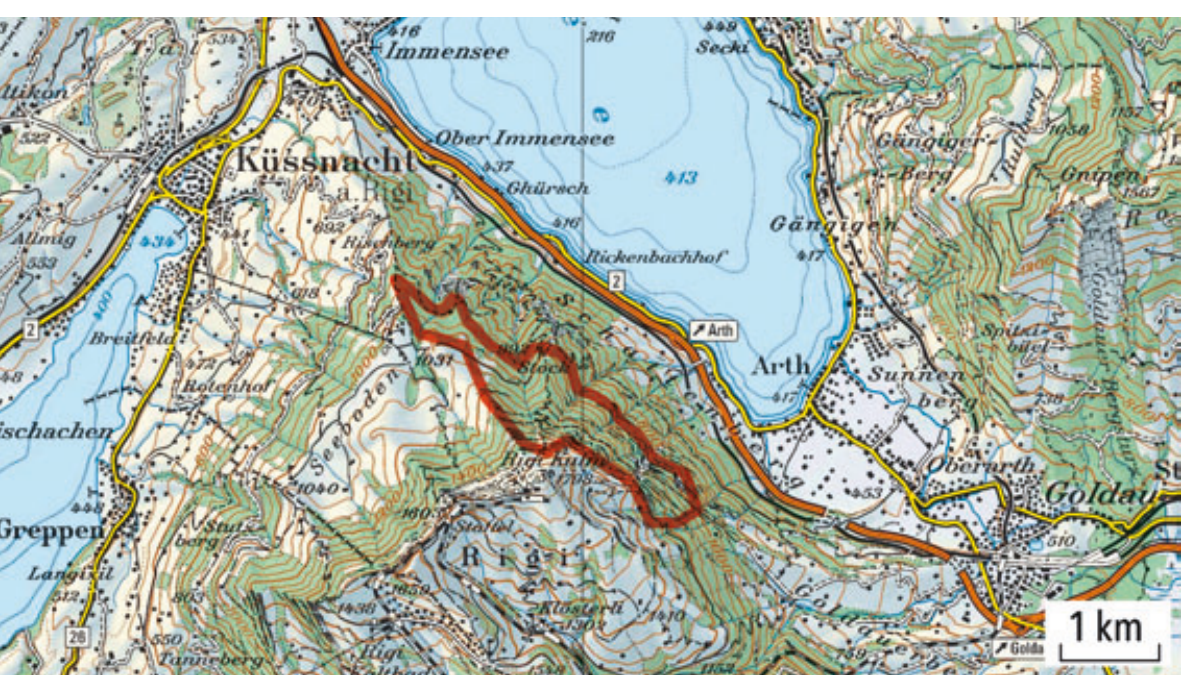

Abb 1 Das Untersuchungsgebiet (rot umrandet) an der Rigi-Nordlehne. Ausschnitt aus der Landeskarte 1:100 000. Reproduziert mit Bewilligung von Swisstopo (BA100435).

Aus diesem Grund wurde 2009 im Rahmen einer Masterarbeit des Studienganges Wald- und Landschaftsmanagement der ETH am Fallbeispiel Rigi-Nordlehne (Kanton Schwyz) der Frage nachgegangen, welche Kosten für die Schutzwaldbewirtschafter durch hohen Verbissdruck entstehen können. Dafür wurde untersucht, mit welchen Kosten an der Rigi-Nordlehne für den Ersatz verloren gegangener Schutzwirkung des Waldes sowie die Erreichung des gemäss NaiS erforderlichen Tannenanteils als wichtiger Komponente einer effizienten Bereitstellung der Schutzwirkung in den nächsten 50 Jahren gerechnet werden muss. Berücksichtigt wurden zukünftige grosse Sturmschadenereignisse, forstliche und jagdliche Massnahmen zur Verminderung des Verbissdruckes sowie Schutzbauten zum Schutz des Waldes vor Schneebewegungen. Die Arbeit stellt die schwierig zu erfassenden ökonomischen Auswirkungen von Verbissschäden in finanziellen Grössen dar. Dies dürfte in der schweizerischen Wald-WildDiskussion eine interessante Ergänzung sein, auch wenn es sich bei den Ergebnissen um Abschätzungen handelt und diese nur anhand eines einzigen konkreten Fallbeispiels hergeleitet worden sind. Der vorliegende Artikel fasst diese Masterarbeit zusammen mit dem Ziel, einen ökonomischen Aspekt in die Wald-Wild-Diskussion um Schweizer Schutzwälder einzubringen.

\section{Untersuchungsgebiet sowie themati- sche und zeitliche Eingrenzung}

Als Untersuchungsgebiet wurde der TannenBuchen-Wald an der Rigi-Nordlehne gewählt (Abbildung 1). Dort stellt sich seit Jahrzehnten die Frage nach einer effizienten Schutzwaldbewirtschaftung: Bereits in den 1980er-Jahren stellten die Bewirtschafter fest, dass die Waldungen überaltert und Massnah- men zur Einleitung der Verjüngung dringend nötig sind, dass aber eine standortgerechte Verjüngung in der Tannen-Buchen-Waldstufe wegen des hohen Verbissdruckes erheblich erschwert und teilweise verunmöglicht wird. Trotz grossen Anstrengungen auf der forstlichen und jagdlichen Seite konnte die Verjüngungssituation im Tannen-Buchen-Wald bisher nicht erheblich verbessert werden (Stadelmann 2008). Dies hat zur Folge, dass die Verjüngung in den letzten 20 Jahren mit grossem finanziellem Aufwand geschützt und punktuell auch Verbauwerke errichtet wurden, um die Schutzwirkung zu erhalten. Zudem haben verschiedene Sturmereignisse gezeigt, dass die Wiederbewaldung von Sturmschadenflächen ohne Vorverjüngung nur sehr zögerlich und nicht mit den gewünschten Baumarten stattfindet, weshalb zusätzliche unterstützende Massnahmen nötig werden. Die Ansprüche an die Wälder an der RigiNordlehne sind hoch: Einerseits sollen sie wichtige Verkehrs- und Versorgungsanlagen, so die SBB-Linie Luzern-Gotthard, die Nationalstrasse A4, die Kantonsstrasse und eine Starkstromleitung sowie diverse Liegenschaften vor Naturgefahren schützen. Steinschlag, Murgang und Schneebewegungen sind dabei die Hauptgefahrenprozesse. Die geologischen Voraussetzungen führen dazu, dass die ganze Nordlehne stark steinschlaggefährdet ist. Entwässert wird das Gebiet von zahlreichen Wildbächen, welche wegen des grossen Pauschalgefälles fast alle ein hohes Murgangpotenzial aufweisen. Sowohl Steinschlagwie auch Erosionsprozesse tragen dabei erheblich zur Geschiebebildung bei. Lawinen und Schneegleiten sind insofern ein Problem, als sie im Bereich der oberen Waldgrenze bedeutende Waldschäden verursachen und die Verjüngung erheblich behindern können.

Andererseits sollen die Wälder an der RigiNordlehne den Wildtieren einen vielfältigen, naturnahen Lebensraum bieten und Ausweichraum schaffen für die touristisch und landwirtschaftlich stark genutzten Areale an der Rigi-Südseite. Das Untersuchungsgebiet befindet sich im kantonsübergreifenden Wildraum 2, welcher nebst der Rigi-Nordlehne auch deren Südseite bis zum Vierwaldstättersee sowie das Gebiet zwischen Goldau, Brunnen, Gersau und Vitznau umfasst. Der Wildbestand an der RigiNordlehne kann somit nicht isoliert betrachtet, sondern muss im Kontext des gesamten Wildraums beurteilt werden. Zwischen 1996 und 2002 hat der Bestand der Gämse - als Hauptverantwortliche für die Verbissprobleme - leicht abgenommen, 2007 war er wieder auf dem Stand von 1996. ${ }^{1}$

\footnotetext{
GRAF RF, BÄCHTIGER M, REMPFLER T, ROBIN K (2008) Streckenanalyse Schalenwild im Gebiet Rigi (Wildraum 2). Wädenswil: Zürcher Hochschule für angewandte Wissenschaften, Fachstelle Wildtier- und Landschaftsmanagement, unveröffentlicht.
} 
In der Masterarbeit, welche diesem Artikel zugrunde liegt, wurde der zeitliche Rahmen auf 50 Jahre festgelegt. Dabei musste ein Kompromiss gefunden werden zwischen einem sehr langen Zeithorizont, wie er für die Betrachtung der langsamen Waldentwicklung geeignet wäre, und einem kürzeren, wie er zur Vermeidung von zu grossen Unsicherheiten bei ökonomischen Fragestellungen zu empfehlen ist.

Als relevante Naturgefahrenprozesse wurden Schneebewegungen sowie Rutschungen und Erosion als Geschiebelieferanten für Murgänge berücksichtigt. Steinschlag wurde insofern miteinbezogen, als dadurch die Machbarkeit verschiedener Massnahmen eingeschränkt wird. In Bezug auf die Wildsituation wurden lediglich die Gämsbestände berücksichtigt, da aufgrund der lokalen Verhältnisse davon ausgegangen wurde, dass Reh und Hirsch in Bezug auf den Verbiss im betrachteten Tannen-BuchenWald eine untergeordnete Rolle spielen.

\section{Methoden}

Die Beantwortung der komplexen Fragestellung verlangte ein differenziertes Vorgehen. Als methodischer Rahmen wurde die Kosten-Wirksamkeits-Analyse (KWA) gewählt, welche eine der am häufigsten angewendeten Methoden zur Beurteilung von Projekten darstellt, in welchen nicht ohne Weiteres alle Wirkungen monetarisiert werden können oder sollen (Hanusch 1994). Als Grundlage für die KWA diente eine ausführliche Situationsanalyse.

Die KWA beinhaltet acht Bearbeitungsschritte (Abbildung 2). Im Folgenden wird dargestellt, wie bei der Erarbeitung der acht Schritte vorgegangen wurde.

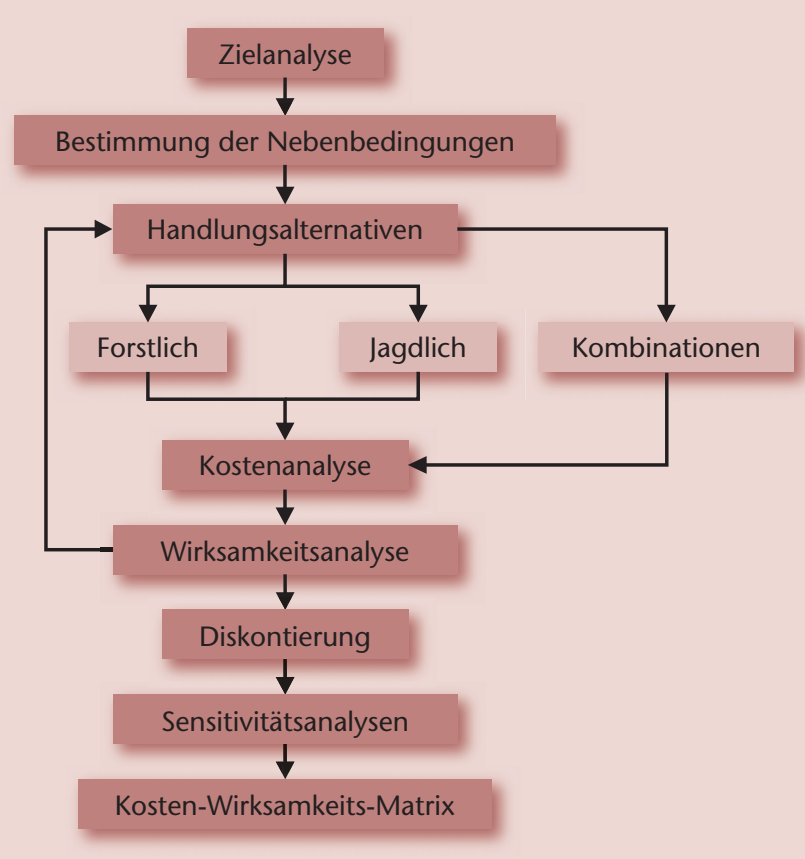

Abb 2 Vorgehen bei der Kosten-Wirksamkeits-Analyse.
Für die Zielanalyse wurde ein problemorientierter Ansatz gewählt: Aufgrund der Erkenntnis, dass an der Rigi-Nordlehne der Verbissdruck so gross ist, dass die Verjüngung der Tanne nicht aufwachsen kann und die Buchen- und Ahornverjüngung beeinträchtigt wird, wurden ein waldbauliches Oberziel und waldbauliche Teilziele gemäss NaiS formuliert, welche durch Handlungsalternativen erreicht werden sollen. Als Oberziel wurde festgelegt, dass die Schutzwirkung gegen gravitative Naturgefahren dauerhaft gewährleistet ist. Teilziele sind eine genügende Verjüngung im Aufwuchs, eine standortgerechte Baumartenmischung in der Verjüngung, keine grossen Lücken ohne Verjüngung und die Begrenzung der Waldschäden durch Waldlawinen.

Als Nebenbedingungen wurden Ansprüche der Wildtierökologie (Erhaltung vitaler Wildpopulationen) sowie jagdliche (Patentjagdsystem) und gesetzliche (Waldgesetz, Jagdgesetz) Rahmenbedingungen eingesetzt.

Die grösste Schwierigkeit stellte die Bestimmung der Handlungsalternativen dar: Als Grundlage für eine Kostenschätzung musste einerseits festgelegt werden, mit welchen forstlich-technischen Massnahmen in den nächsten 50 Jahren gerechnet werden muss, um die waldbaulichen Ziele an der Rigi zu erreichen. Da eine flächendeckende gutachtliche Beurteilung aus zeitlichen Gründen nicht möglich war, wurde dieser Schritt GIS-basiert ausgeführt. Dabei wurde auf das Waldentwicklungsmodell Rigfor zurückgegriffen. Darin wird die Waldentwicklung unter aktuellem und reduziertem Verbissdruck simuliert (Stadelmann 2011, dieses Heft). Zusätzlich wurde Rigfor durch eine Simulierung von grossen Sturmschadenflächen (durchschnittliche Grösse 1.55 ha) ${ }^{2}$ sowie eine Prognostizierung der Wiederbewaldung derselben unter aktuellem und reduziertem Verbissdruck ergänzt. Anhand der so prognostizierten Waldzustände wurden für verschiedene Zeitpunkte kritische Stellen ausgewählt, an welchen gemäss NaiS die Schutzfunktion nicht erfüllt wird. Die mit dem Szenario «aktueller Verbissdruck» modellierten Lücken- und Jungwuchsflächen wurden als minimal zu erwartende Lücken- und Jungwuchsflächen betrachtet, da in Rigfor die Lückenfläche unter dem Szenario «aktueller Verbissdruck» unterschätzt wird (Stadelmann 2011, dieses Heft). Anschliessend wurde wiederum GIS-basiert bestimmt, welche Massnahmen an dieser Stelle aufgrund der lokalen Bedingungen (Geländeneigung, Steinschlaggefährdung und Grösse der zu behandelnden Fläche) machbar und zielführend sind.

\footnotetext{
2 Flächengrösse wie beim Hagelsturm im Jahr 1992 und beim Sturm Lothar (1999) an der Rigi-Nordlehne. Die Flächen wurden aufgrund der Sturmanfälligkeit (Alter, Bestandesaufbau, Baumarten) ausgewählt.
} 
Nebst dieser Herleitung der forstlichen Massnahmen musste zudem bestimmt werden, welche jagdlichen Massnahmen als Grundlage für eine Kostenschätzung dienen sollen. Dazu wurde anhand aktueller Wildbestandsschätzungen ein Zielbestand festgelegt und mithilfe von Literaturangaben und Expertenbefragungen der zeitliche Aufwand abgeschätzt, mit welchem zu rechnen ist, um diesen Zielbestand über 50 Jahre mittels Schwerpunktbejagung in ausgewählten Flächen zu erhalten.

Die so bestimmten Massnahmen bildeten die Grundlagen für die Kostenschätzung, welche mithilfe von Pauschalansätzen erfolgte (für die genauen Annahmen siehe Gasser 2009: 56 ff.).

Die nächste Herausforderung stellte die Wirksamkeitsanalyse dar. Sowohl die Wirkung von Verbissschutzmassnahmen wie auch die Auswirkungen einer Wildbestandsregulierung auf die Verbisssituation sind schwierig zu bestimmen. Grundsätzlich wurden die Massnahmen so ausgelegt, dass entsprechend heutigem Wissen die waldbaulichen Ziele gemäss NaiS erreicht werden können, womit sich im Prinzip die Wirksamkeitsanalyse erübrigte. Im Laufe der Ausarbeitung der zu ergreifenden Massnahmen wurde aufgrund der Simulationen (bei den forstlichen Massnahmen) und Diskussionen mit Experten (bei den jagdlichen Massnahmen) aber klar, dass weder mit rein forstlichen noch mit rein jagdlichen Massnahmen die waldbaulichen Ziele gemäss NaiS erreicht werden können (siehe Resultate). Deshalb wurde zusätzlich eine Kombination von forstlichen und jagdlichen Massnahmen vorgeschlagen, für welche davon ausgegangen wird, dass die geforderte Wirkung erreicht werden kann.

Für die Diskontierung wurden die berechneten Kosten mit einem Realzinssatz von 2.6\% abgezinst. Durch diese zeitliche Homogenisierung werden Kosten, die zu unterschiedlichen Zeitpunkten anfallen, vergleichbar gemacht (Bergen et al 2002). Damit wird der Tatsache Rechnung getragen, dass einerseits nicht alle Kosten einer Massnahme zum gleichen Zeitpunkt anfallen (z. B. Investitionskosten und Unterhaltskosten) und andererseits die verschiedenen Massnahmen sehr unterschiedliche Investitionszeitpunkte aufweisen.

Den zahlreichen Unsicherheiten, welche sich teilweise bereits durch die Fragestellung mit ihrem starken Bezug auf zukünftige Zustände ergeben, wurde durch Sensitivitätsanalysen Rechnung getragen. Dabei wurde untersucht, welchen Einfluss die Annahmen bezüglich des Diskontierungssatzes sowie des Aufwandes für die jagdlichen Massnahmen auf die Endbewertung der verschiedenen Massnahmenvarianten haben. Schliesslich wurde anhand der Kosten-Wirksamkeits-Matrix eine ökonomische Bewertung der vorgeschlagenen Massnahmen vorgenommen.

\section{Resultate}

Im Folgenden werden die wichtigsten Resultate aus der Masterarbeit präsentiert. Die ausführlichen Ergebnisse und die Berechnungsgrundlagen finden sich in Gasser (2009).

\section{Tannenanteil und Schutzwirkung}

Die Tanne ist nicht nur aus waldbaulichen, sondern auch aus ökonomischen Gründen im heutigen Schutzwald an der Rigi-Nordlehne sehr vorteilhaft. Anhand der prognostizierten Entwicklung von Sturmschadenflächen konnte gezeigt werden, dass bei einem Rückgang des Tannenanteils die Schutzwirkung auf der Gesamtfläche der Rigi-Nordlehne langfristig stark zurückgeht. Dies deshalb, weil bei der Wiederbewaldung kaum auf Vorverjüngung der Tanne gesetzt werden kann und somit die Lücken sehr viel länger in einem nicht schutzwirksamen Zustand verbleiben, als wenn sich die Verjüngung bereits vor dem Ereignis hätte etablieren können. Daraus resultiert insgesamt eine grössere nicht schutzwirksame Fläche, als wenn bereits Vorverjüngung vorhanden wäre. Um die Schutzwirkung auch ohne die Tanne erhalten zu können, müssen auf diesen Flächen kostenintensive Massnahmen (z. B. Fichtenpflanzungen, Bekämpfung der Vegetationskonkurrenz) zur Unterstützung und Förderung der Verjüngung ergriffen werden.

\section{Wirksamkeit der Handlungsalternativen}

Weder mit rein forstlichen noch mit rein jagdlichen Massnahmen kann an der Rigi-Nordlehne eine flächendeckende Verjüngung mit dem in Nais verlangten Tannenanteil erreicht werden.

Bei der Handlungsalternative mit rein forstlichen Massnahmen zeigen sich verschiedene Schwierigkeiten: So sind in den nächsten 50 Jahren insgesamt 130 ha vor Verbiss zu schützen, was knapp 70\% der Waldfläche in der Tannen-Buchen-Waldstufe entspricht. Auf gegen 10\% der zu schützenden Fläche ist es wegen der lokalen Voraussetzungen (Hangneigung, Steinschlag etc.) nicht möglich, Verbissschutzmassnahmen zu ergreifen, wobei ein Grossteil der nicht schützbaren Flächen im Einzugsgebiet des Langweidbachs liegt, welcher als murfähig gilt, weshalb eine schutzwirksame Bestockung besonders wichtig wäre. Gegen 7\% der Fläche sollen mit Zäunen geschützt werden, eine Massnahme, die an der RigiNordlehne insbesondere wegen Steinschlag erfahrungsgemäss nicht genügend wirksam ist. 70 bis $90 \%$ der zu schützenden Fläche müssen mit chemischem Verbissschutz behandelt werden. Um grosse Flächen über einen genügend langen Zeitraum konsequent mit solchen Mitteln zu behandeln, ist grosser Einsatz seitens der Ausführenden erforderlich. Der Erfolg dieser Massnahme steht und fällt daher mit der Motivation und Zuverlässigkeit der Ausführenden. 
Rein forstliche Massnahmen

Rein jagdliche Massnahmen (mässige Bestandesreduktion)

Rein jagdliche Massnahmen (starke Bestandesreduktion)

Kombination I

(jagdlich und forstlich)

Kombination II

(jagdlich und forstlich)

Kombination II

(jagdlich und forstlich,

zehn Jahre später)

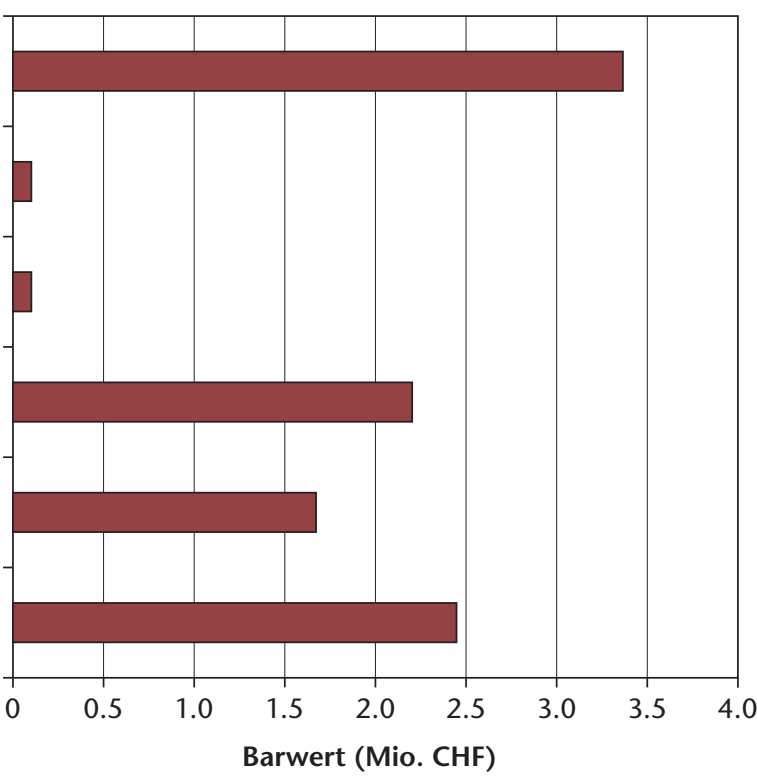

Abb 3 Kostenschätzungen für rein forstliche, rein jagdliche sowie für die Kombination von jagdlichen und forstlichen Massnahmen. Dabei ist zu beachten, dass die rein forstlichen und die rein jagdlichen Massnahmen keine genügende Wirksamkeit aufweisen.

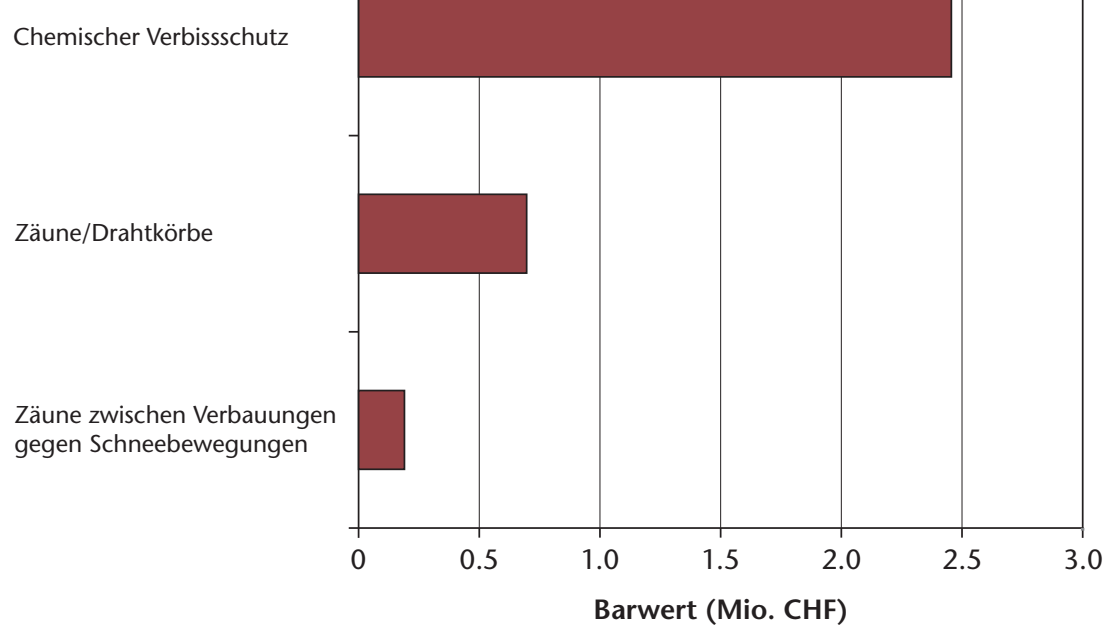

Abb 4 Aufteilung der rein forstlichen Kosten von total 3.3 Mio. Schweizer Franken auf die verschiedenen Verbissschutzmassnahmen.

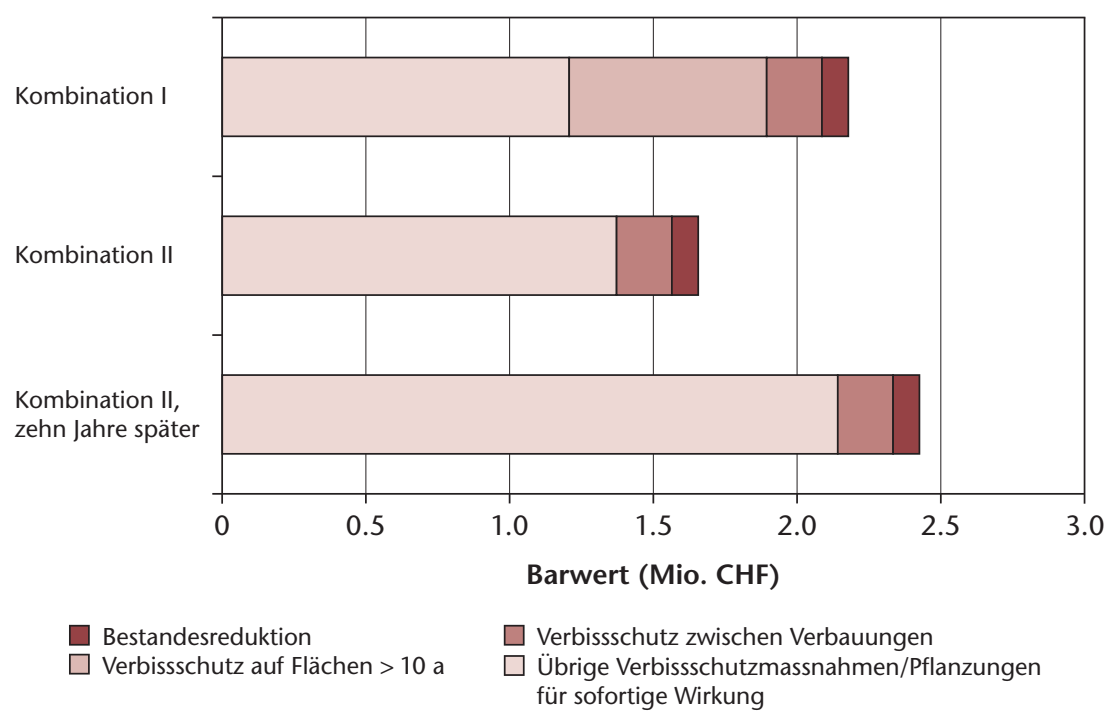

Abb 5 Aufteilung der Kosten bei den Massnahmenkombinationen auf jagdliche und forstliche Massnahmen.
Aus diesen Gründen sind rein forstliche Massnahmen, ganz abgesehen von den hohen Kosten, nicht genügend wirksam und deshalb nur begrenzt sinnvoll.

Bei der Handlungsalternative mit rein jagdlichen Massnahmen zeigt sich, dass mit einer schwerpunktmässigen Bestandesreduktion an der RigiNordlehne der Verbissdruck insgesamt stark reduziert werden kann, da die Zuwanderung von der Südseite nach einer Bestandesregulierung auf der Nordseite dank der Standorttreue der Waldgämse verzögert ablaufen dürfte und der Wechsel der Gämsen von der Süd- auf die Nordseite ohnehin nicht sehr intensiv zu sein scheint. Aufgrund der Beliebtheit der Tanne bei der Gämse sowie wegen der Empfindlichkeit der Tanne hinsichtlich Verbiss muss aber dennoch davon ausgegangen werden, dass die Tannenverjüngung lokal durch Verbiss beeinträchtigt werden dürfte. Das bedeutet, dass auch rein jagdliche Massnahmen nicht ausreichen, um den Tannenanteil gemäss NaiS zu erreichen.

Angesichts dieser Erkenntnisse wurden zusätzlich die Kosten für zwei Massnahmenkombinationen abgeschätzt, welche nach heutigem Kenntnisstand als genügend wirksam beurteilt werden.

\section{Kosten der Handlungsalternativen}

In den nächsten 30 bis 50 Jahren entstehen an der Rigi-Nordlehne folgende Kosten (Barwert; Abbildung 3):

- Circa 3.3 Mio. Schweizer Franken für rein forstliche Massnahmen, falls die Verbissprobleme trotz der ungenügenden Wirksamkeit mit rein forstlichen Massnahmen gelöst werden sollen. Als Grundlage für die Kostenschätzung wurden Wildschutzzäune und Drahtkörbe (dort, wo dies aufgrund der Steinschlaggefährdung möglich ist) sowie chemischer Verbissschutz (wo dies angesichts der Zugänglichkeit und der Hangneigung möglich und zumutbar ist) eingerechnet. Die Aufteilung der Kosten auf die verschiedenen Verbissschutzmassnahmen ist in Abbildung 4 dargestellt; der genaue Umfang der zugrunde liegenden Massnahmen ist in Gasser (2009: $115 \mathrm{ff}$.) enthalten.

- Circa 87000 bis 90000 Schweizer Franken für eine rein jagdliche Lösung der Verbissprobleme: Eine Reduktion des Gämsbestands an der Rigi-Nordlehne und eine Erhaltung desselben auf einem tiefen Niveau käme auf gut 87000 Schweizer Franken (Reduktion um 20\%) bis gut 90000 Schweizer Franken (massive Reduktion um 50\%) während der nächsten 50 Jahren zu stehen, wobei davon ausgegangen wird, dass die Hälfte der Abschüsse durch Jäger, die andere Hälfte durch professionelle Wildhüter in Form von Hegeabschüssen getätigt werden (vgl. Gasser 2009: $131 \mathrm{ff}$.$) .$

- $\quad 1.5$ bis 2.2 Mio. Schweizer Franken für eine als ausreichend wirksam beurteilte Kombination von 
forstlichen und jagdlichen Massnahmen: Dabei enthalten beide Kombinationen Verbissschutzmassnahmen inklusive Pflanzungen zwischen den Verbauungen und auf aktuell grossen Lückenflächen. Kombination I umfasst zudem eine Reduktion des Gämsbestandes um 20\% und Verbissschutzmassnahmen/Pflanzungen auf Lückenflächen grösser als 10 a, Kombination II eine Reduktion des Gämsbestandes um 50\%. Wird die Kombination II um zehn Jahre hinausgeschoben, entstehen dadurch Mehrkosten von 800000 Schweizer Franken (Abbildung 5).

- Circa 3 Mio. Schweizer Franken für Verbauungen gegen Schneebewegungen: Mit diesen Kosten muss unabhängig von der Frage, wie sich der Verbissdruck in den kommenden Jahren entwickeln wird, gerechnet werden. Einerseits müssen Schäden am Schutzwald durch Waldlawinen verhindert werden, andererseits muss die Verjüngung vor Schneebewegungen geschützt werden. Insgesamt weisen in den nächsten 50 Jahren gut 7 ha des Schutzwaldes eine ungenügende Schutzwirkung auf und müssen deshalb mit Dreibeinböcken oder temporären Schneerechen verbaut werden.

\section{Diskussion}

\section{Tannenanteil}

Die ökonomischen Vorteile der Tanne im heute vorhandenen Schutzwald an der Rigi-Nordlehne wurden in dieser Arbeit aufgezeigt. Dies ist zwar grundsätzlich keine neue Erkenntnis, aber ein wichtiges Argument für den in NaiS für eine effiziente Schutzwaldbewirtschaftung geforderten Tannenanteil. Was aber in dieser Studie nicht beantwortet wird, ist die Frage, ob die Kosten für die Erreichung des Tannenanteils an der Rigi-Nordlehne tiefer sind als die Kosten für Massnahmen zur Erreichung der Schutzwirksamkeit mit anderen Baumarten. Hier besteht weiterer Forschungsbedarf.

\section{Forstliche Massnahmen und Verbauungen}

Die Kosten für die Verbauungen gegen Schneebewegungen liegen in einem realistischen Rahmen. Sie sind teilweise auf die Verbisssituation der letzten Jahrzehnte zurückzuführen und hätten mit einer Verbesserung derselben in der Vergangenheit vermieden werden können. Auch die Resultate bezüglich der Verbissschutzmassnahmen, der damit erreichten Wirksamkeit und der dafür entstehenden Kosten werden insgesamt als plausibel beurteilt.

Allerdings wurden die forstlichen Massnahmen rein GIS-basiert bestimmt und konnten aus zeitlichen Gründen nicht im Feld überprüft werden. Eine erst nach Abschluss der Masterarbeit durchgeführte Begehung zeigte aber, dass diejenigen Flächen, welche zu Beginn des Betrachtungshorizontes für Massnahmen gegen Schneebewegungen ausgeschieden worden waren, heute tatsächlich eine ungenügende Schutzwirksamkeit aufweisen. Mindestens diesbezüglich scheint eine GIS-basierte Ausscheidung der kritischen Flächen zweckmässig.

Bei den Kosten für Massnahmen gegen Schneebewegungen und für Verbissschutz ist davon auszugehen, dass es sich dabei eher um eine Kostenuntergrenze handelt und der tatsächliche Betrag noch höher ausfallen dürfte (siehe Kapitel «Methoden»). Es handelt sich bei diesen Kosten ausdrücklich um Abschätzungen, welche auf plausiblen Annahmen bezüglich eines zukünftigen Waldzustandes und auf Pauschalansätzen beruhen. Solche Voraussagen sind mit vielen Unsicherheiten verbunden und sollten daher lediglich als grobe Kenngrössen verstanden werden.

\section{Jagdliche Massnahmen}

Die Kostenschätzungen sowie die Beurteilung der Wirksamkeit für die jagdlichen Massnahmen scheinen grundsätzlich ebenfalls realistisch. Allerdings ist zu beachten, dass die Unterschiede in der Kostenschätzung zwischen mittlerer und starker Bestandsreduktion im Bereich der Unsicherheit liegen und somit vernachlässigt werden können.

Die Kostenschätzungen basieren auf Wildbestandsschätzungen für den gesamten Wildraum 2 . Diese Wildbestandsschätzungen wurden aufgrund der Zählergebnisse auf die Nordlehne reduziert. Dabei wurden die Nord-Süd-Bewegungen berücksichtigt, soweit dies mit den vorliegenden Daten möglich war. Trotzdem sind die Wildbestandsschätzung und somit auch der gewählte Zielbestand mit grossen Unsicherheiten behaftet. Aus den durchgeführten Sensitivitätsanalysen wurde aber deutlich, dass sich zwar die absoluten Beträge für die jagdlichen Massnahmen bei unterschiedlichen Annahmen deutlich ändern können, die Beurteilung und Einordnung der Massnahmenvarianten aber gleich bleiben. Somit ist den Unsicherheiten bezüglich der Wildbestandsgrösse in der Frage der ökonomischen Beurteilung der Massnahmenvarianten ein geringes Gewicht zuzuordnen.

Eine Schwerpunktbejagung der Gämsbestände an der Rigi-Nordlehne ist nicht einfach, und es braucht einen grossen Einsatz der Jägerschaft, um eine solche erfolgreich umzusetzen. Falls die Bereitschaft dazu fehlt, müssten allenfalls professionelle Wildhüter eingesetzt werden.

Nebst der Wildbestandsreduktion kommen grundsätzlich auch weitere Massnahmen wie Lebensraumaufwertungen oder Wildruhezonen auf der Südseite der Rigi infrage. Im Rahmen der vorliegenden Studie wurde aber darauf verzichtet, Kostenschätzungen für weitere Massnahmen durchzuführen. 


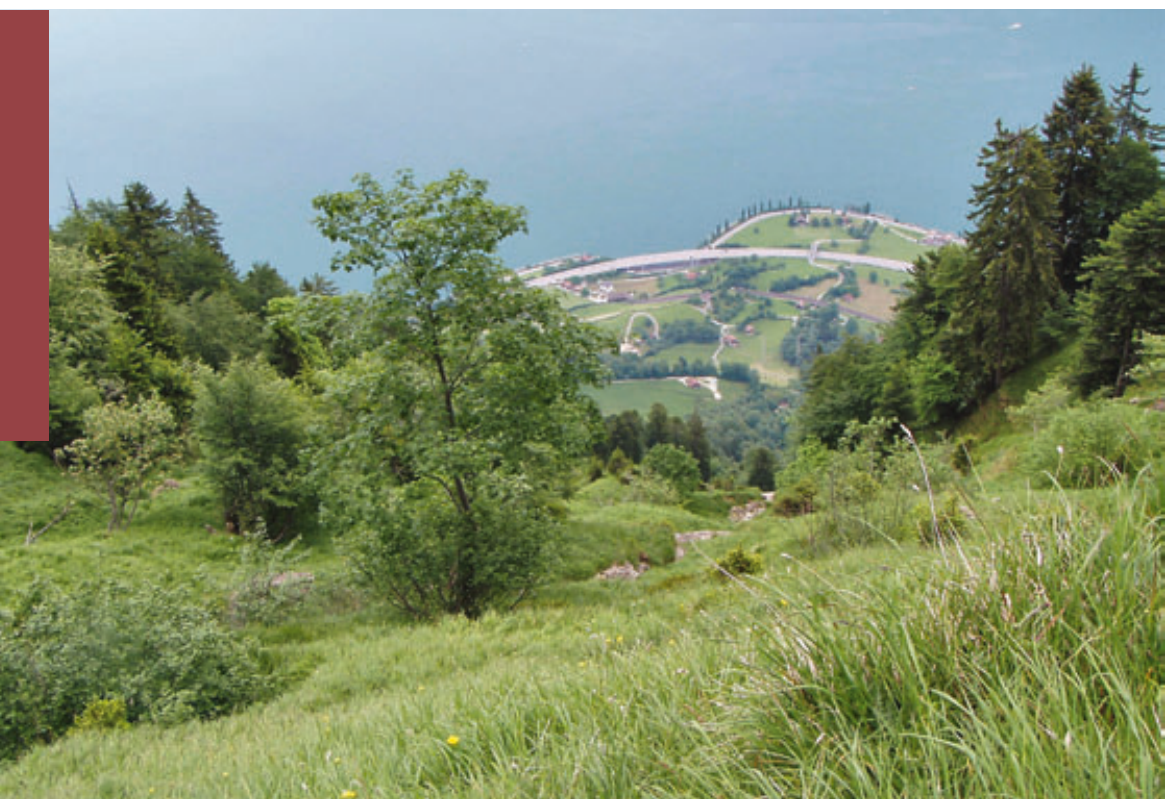

Abb 6 Blick von der Rigi-Nordlehne auf das Schadenpotenzial.

\section{Massnahmenkombinationen}

Die Massnahmenkombinationen werden grundsätzlich als wirksam und realistisch beurteilt. Es ist aber schwierig abzuschätzen, wie viel Verbissschutzmassnahmen nach einer Bestandsreduktion noch nötig sind. Die Kostenschätzungen dürften sich eher im oberen Bereich bewegen, und es darf angenommen werden, dass mit einer massiven Bestandsreduktion eher weniger Aufwendungen für Verbissschutzmassnahmen nötig werden als geschätzt.

\section{Verwendete Methode}

Der Vergleich von so grundsätzlich verschiedenen Massnahmen wie Verbissschutz und jagdlichen Eingriffen ist sehr schwierig: Die Massnahmen erzielen unterschiedliche Wirkungen, weisen unterschiedliche Investitionszeitpunkte auf und basieren auf zahlreichen Annahmen. Die Kosten-Wirksamkeits-Analyse als methodischer Rahmen hat sich grundsätzlich für diese Art Problemstellung als geeignet herausgestellt, weil den unterschiedlichen Wirkungen durch die Berechnung von Kosten-Wirksamkeits-Faktoren Rechnung getragen wird, die zu unterschiedlichen Zeitpunkten getätigten Investitionen durch Diskontierung vergleichbar gemacht werden und den Unsicherheiten bei den Annahmen mit Sensitivitätsanalysen begegnet wird.

Insbesondere die Beurteilung der Wirkungen bleibt aber in der vorliegenden Arbeit sehr schwierig, nicht zuletzt wegen des weiten Zeithorizonts. Der 50 Jahre umfassende Betrachtungszeitraum ist einerseits notwendig, um der langsamen Waldentwicklung gerecht zu werden; andererseits führt er dazu, dass durch die Diskontierung die Kosten von relativ spät anfallenden Massnahmen nur noch geringen Einfluss auf das Gesamtergebnis haben. Entscheidungsträger könnten sich dadurch veranlasst sehen, Schutzmassnahmen und die damit verbundenen Kosten aufzuschieben und künftigen Generationen zu übertragen. Die Berechnungen für die um zehn Jahre hinausgeschobene Massnahmenkombination II zeigen jedoch eindrücklich, dass durch schnelles Handeln ein Kostenanstieg vermieden werden kann.

Sensitivitätsanalysen tragen dazu bei, die Auswirkungen von Unsicherheiten besser erkennen zu können (vgl. Gasser 2009: 156). Dennoch bleibt zu bedenken, dass sich bei der vorliegenden Untersuchung die Fragestellung zu einem grossen Teil auf zukünftige Zustände bezieht und somit sehr viele Annahmen erforderlich sind, die die Resultate entscheidend beeinflussen. Es ist jedoch festzustellen, dass bei der Herleitung der Annahmen grundsätzlich sehr zurückhaltend vorgegangen wurde.

\section{Schlussfolgerungen}

Wegen der grossen Verbissprobleme entstehen für die Waldbewirtschafter an der Rigi-Nordlehne erhebliche Kosten, um die Baumartenmischung gemäss NaiS langfristig zu erhalten. Alle Kosten, sowohl diejenigen für Verbauungen gegen Schneebewegungen als auch diejenigen für Verbissschutzmassnahmen, wären deutlich geringer, wenn die Verbisssituation in den vergangenen 20 Jahren verbessert worden wäre. Ein Teil der Kosten fällt unabhängig von der künftigen Entwicklung der Verbisssituation an, ein bedeutender Teil könnte aber durch eine Reduktion des Verbissdruckes vermieden werden.

Ein Lösungsansatz mit rein forstlichen Massnahmen verursacht nicht nur die höchsten Kosten, sondern ist auch mit einer ungenügenden Wirkung verbunden. Eine Erreichung der waldbaulichen Ziele ohne massive jagdliche Eingriffe scheint somit kaum möglich. Aber auch ein Ansatz mit rein jagdlichen Massnahmen dürfte nicht den gewünschten Erfolg bringen. Zur umfassenden Lösung der Verjüngungsprobleme an der Rigi-Nordlehne wird daher eine Kombination von forstlichen und jagdlichen Massnahmen empfohlen. Dabei müssen jagdliche Massnahmen dafür sorgen, dass insgesamt das Verhältnis von abgebissenen zu vorhandenen Tannen nicht mehr zu einer Stammzahlreduktion der Tanne führt. Ziel der forstlich-technischen Massnahmen muss es sein, punktuell Flächen mit nach wie vor verbissbedingten Verjüngungsschwierigkeiten zu schützen.

Neu an den Ergebnissen dieser Arbeit ist, dass die durch den hohen Verbissdruck entstehenden ökonomischen Nachteile quantifiziert werden und somit ein direkter Kostenvergleich verschiedener Massnahmenvarianten ermöglicht wird. Diese Massnahmen müssen mindestens teilweise mit öffentlichen Geldern finanziert werden. Deshalb wäre es aus volkswirtschaftlicher Sicht wünschenswert, 
wenn im Hinblick auf einen effizienten Umgang mit öffentlichen Geldern für die Naturgefahrenabwehr und insbesondere die Schutzwaldbewirtschaftung (Abbildung 6) auch in der Wald-Wild-Frage vermehrt nach wirtschaftlich effizienten Lösungen gesucht würde.

Eingereicht: 16. August 2010, akzeptiert (mit Review): 23. November 2010

\section{Literatur}

BERGEN V, LÖWENSTEIN W, OLSCHEWSKI R (2002) Forstökonomie - Volkswirtschaftliche Grundlagen. München: Vahlen. $469 \mathrm{p}$.

BRASSEL P, BRÄNDLI UB, EDITORS (1999) Schweizerisches Landesforstinventar. Ergebnisse der Zweitaufnahme 1993-1995. Bern: Haupt. 442 p.

FREHNER M, WASSER B, SCHWITTER R (2005) Nachhaltigkeit und Erfolgskontrolle im Schutzwald. Wegleitung für Pflegemassnahmen in Wäldern mit Schutzfunktion. Bern: Bundesamt Umwelt Wald Landschaft. 564 p.

\section{Ökonomische Konsequenzen der Verbiss- probleme an der Rigi-Nordlehne}

In der Schutzwaldbewirtschaftung spielt die Verjüngung mit geeigneten Baumarten eine entscheidende Rolle. Diese wird in vielen Regionen der Schweiz durch starken Wildverbiss erschwert; insbesondere die Verjüngung der waldbaulich wertvollen Weisstanne wird stellenweise stark beeinträchtigt. Dies kann für die Waldbewirtschafter zu hohen Kosten für die Unterstützung der Verjüngung und den Ersatz verloren gegangener Schutzwirkungen führen. Mit einer Kosten-Wirksamkeits-Analyse und auf der Basis des Waldentwicklungsmodells Rigfor wurde am Beispiel der Rigi-Nordlehne untersucht, 1) mit welchen Kosten in den nächsten 50 Jahren für Verbissschutzmassnahmen gerechnet werden muss, wenn der Verbissdruck nicht bedeutend gesenkt werden kann, 2) welche Kosten für eine Reduktion des Verbissdruckes durch jagdliche Massnahmen und 3) welche für Verbauungen gegen Schneebewegungen zu erwarten sind. Resultat der Studie sind Kostenkalkulationen für rein forstlich-technische, rein jagdliche und kombinierte Massnahmen sowie für notwendige Verbauungen zum Schutz des Waldes gegen Schneebewegungen. Es zeigt sich, dass sich der hohe Verbissdruck an der RigiNordlehne nicht nur aus waldbaulicher Sicht nachteilig auswirkt, sondern er auch negative ökonomische Konsequenzen hat. Diese belaufen sich je nach Massnahmenvariante auf rund 3 Mio. bis 6.6 Mio. Schweizer Franken in einem Zeitraum von 50 Jahren. Am Beispiel der Rigi-Nordlehne wird damit ein neues Argument in die Diskussion um die Verjüngungssituation eingebracht, welche für viele Schutzwälder der Schweiz seit Jahren hauptsächlich mit waldbaulichen und wildtierökologischen Argumenten geführt wird.
GASSER N (2009) Ökonomische Bewertung von Schutzwaldpflege und technischen Schutzmassnahmen am Beispiel der Rigi-Nordlehne. Zürich: ETH Zürich, Waldökologie, Masterarbeiten. 204 p. doi: 10.3929/ethz-a-005985253

HANUSCH H (1994) Nutzen-Kosten-Analyse. München: Vahlen, 2 ed. 202 p.

ODERMATT O (1999) Einfluss freilebender Wiederkäuer auf die Verjüngung des Schweizer Waldes. Schweiz Z Forstwes 150: 313-326. doi: 10.3188/szf.1999.0313

PLANAT (2005) Strategie Naturgefahren Schweiz. Synthesebericht. Bern: Bundesamt Wasser Geologie, Nationale Plattform Naturgefahren. $88 \mathrm{p}$.

STADELMANN G (2008) Modellierung der Waldentwicklung an der Rigi-Nordlehne zur Analyse der Schutzwirkung von Wald gegen Murgang. Zürich: ETH Zürich, Professur Waldökologie, Masterarbeiten. 79 p. doi: 10.3929/ethza-005968602

STADELMANN G (2011) Modellierung des Einflusses von Wildverbiss auf die Schutzwaldentwicklung an der Rigi-Nordlehne. Schweiz Z Forstwes 162: 355-363. doi: 10.3188/ szf.2011.0355

\section{Conséquences économiques des problèmes d'abroutissement au versant nord du Rigi}

Le rajeunissement d'essences adaptées joue un rôle décisif pour la gestion des forêts protectrices. Dans de nombreuses régions de Suisse, il est cependant rendu difficile par un abroutissement intensif, en particulier le rajeunissement du sapin - une essence précieuse du point de vue sylvicole - est par endroits fortement entravé. Ce problème peut entraîner des coûts élevés pour le gestionnaire, que ce soit pour encourager le rajeunissement d'une part et pour remplacer la fonction protectrice de la forêt perdue d'autre part. Au moyen d'une analyse coût-efficacité et sur la base du modèle de développement forestier Rigfor, les questions suivantes ont été étudiées à l'exemple du versant nord du Rigi: à quels coûts doit-on s'attendre dans les 50 prochaines années 1) pour des mesures de protection contre l'abroutissement si la pression du gibier ne diminue pas significativement, 2) pour une réduction de la pression du gibier par la chasse et 3) pour des ouvrages de protection contre les mouvements du manteau neigeux. Le résultat de cette étude est le calcul des coûts pour les mesures forestières, les mesures de régulation du gibier, les mesures combinées, ainsi que pour les ouvrages nécessaires à prévenir les mouvements du manteau neigeux. II s'avère que sur le versant nord du Rigi, la forte pression du gibier n'a pas seulement des conséquences négatives du point de vue sylvicole, mais également du point de vue économique. Celles-ci s'élèvent de 3 mio à 6.6 mio de francs suisses dans une période de 50 ans dépendant de la variante de mesures choisie. A l'exemple du versant nord du Rigi, un nouvel argument est apporté à la discussion quant à la problématique du rajeunissement dans les forêts protectrices qui, en Suisse, s'articule depuis des années surtout autour de la sylviculture et de l'écologie des animaux sauvages. 